Бармашов Константин Сергеевич канд. экон. наук, ФГБОУ ВО

«Российский экономический университет имени Г.В. Плеханова», г. Москва

e-mail: k.barmashov@ru.faberlic.com

\section{Barmashov Konstantin}

Candidate of Economic Sciences,

Plekhanov Russian University

of Economic, Moscow

e-mail: k.barmashov@ru.faberlic.com

\section{ГАРМОНИЗАЦИЯ ПРОМЫШЛЕННОЙ И ТОРГОВОЙ ПОЛИТИКИ ПРЕДПРИЯТИЯ}

\begin{abstract}
Аннотация. Рассмотрены методологические и методические вопросы определения понятия гармонизации в экономике и оценки гармонизации функиионирования социальноэкономической системы, которые конкретизированы на примере промышленной и торговой политики промышленного предприятия. Исследование вопросов гармонии в экономике раскрывает существуюшие антагонистические противоречия во внешней и внутренней средах деятельности участников экономических отношений. Показано, что гармонизачия промышленной и торговой политик предприятия - это, с одной стороны стратегический план производства и продаж, а с другой стороны, процесс согласования иелей, принципов, методов, критериев и показателей производственных, сбытовых и обеспечивающих их бизнес-процессов на всех этапах жизненного цикла политики, который приводит к снижению экономического ущзерба.

Ключевые слова: гармонизация, промышленное предприятие, промышленная политика, торговая политика, экономическая безопасность.
\end{abstract}

\section{HARMONIZATION OF INDUSTRIAL AND TRADE POLICY OF THE ENTERPRISE}

\begin{abstract}
Methodological and methodical questions of definition of a concept of harmonization in economy and estimates of harmonization of social and economic system functioning, concretized on the example of harmonization of industrial and trade policy of the industrial enterprise are considered. The research of questions of harmony in economy opens the existing antagonistic contradictions in external and internal environments of activity of participants of the economic relations. Harmonization of industrial and trade policy of the enterprise is, on the one hand, the strategic plan of production and sales, and on the other hand, process of coordination of purposes, principles, methods, criteria and indicators of the production, marketing and business processes providing them at all stages of life cycle of policy which leads to decrease of economic damage. Keywords: harmonization, industrial enterprise, industrial policy, trade policy, economic security.
\end{abstract}

Понятие гармонии не так часто используется в экономических исследованиях [8]. В «Толковом словаре» В. Даля гармония рассматривается как созвучие, соответствие, равновесие, соразмерность, равнозвучие, равномерность, взаимность соотношений, согласность, согласие, благостройность, стройность; соразмерные отношения частей целого; правильные отношения современных или одновременных звуков, аккорды; наука о возможных созвучиях [4]. Однако в публикациях в слабой степени исследованы вопросы оценки гармонизации функционирования социально-экономических систем. Этой проблеме посвящена предлагаемая статья, в которой рассмотрены методологические вопросы определения понятия гармонизации в экономике и оценки гармонизации функционирования социально-экономической системы, которые конкретизированы на примере гармонизации промышленной и торговой политики промышленного предприятия.

Вопросы гармонии в экономике или экономических отношениях стали рассматриваться с середины XIX века, и первым трудом в данной сфере была работа «Экономические гармонии» Ф. Бастиа [2]. Ключевая мысль Ф. Бастиа связана с тем фактом, что возможности участников экономических отношении в значительной степени превосходят их нужды (так называемый эффект синергии), в силу этого экономические отношения в полной мере свободны от существующих антагонистических противоречий во внешней и внутренней средах деятельности участников экономических отношений. Участник экономических отношений может быть неэффективным, но в полной мере быть не гармоничным данный участник не может, поскольку это приведет к нерациональному использованию его возможностей. Фактически, именно Ф. Бастиа были заложены основные положения теории гармонизации экономических отношений.

(C) Бармашов К.C., 2018 
В современной науке начала XXI века в России, а также в зарубежных странах закон пропорционального, планомерного развития экономических процессов трансформировался в закон обеспечения пропорциональности, композиции (или закон гармонии) в рамках больших одновременных потерь для развития непосредственно теории гармонии процессов. При этом сегодня нет общепризнанных и целостных положений теории гармонизации. Подобных примеров по реализации основных положений закона гармонизации есть великое множество как для современной экономической теории, так и для теории управления (менеджмента).

Наша трактовка понятия гармонии в экономических отношениях связана с установлением идеального динамичного и/или статичного состояния социально-экономической системы, которое характеризуется пропорциональностью процессов на этапах воспроизводственного цикла (производство, распределение, обмен, потребление), а также упорядоченностью данных процессов, выбранной рациональной моделью специализации системы, максимальной согласованностью и сбалансированностью этих процессов, их соответствием требованиям внешней среды и друг другу по критерию единства социальной, экономической и экологической составляющих деятельности, выраженных соответствующими целевыми установками (системой ключевых показателей) по использованию интеллектуальных и материальных ресурсов системы.

Фактически понятие «гармонии в экономике» - субъективное и идеализированное. При этом в современных социально-экономических условиях стремление быть гармоничным, совершенствоваться (повышать свою эффективность) свойственно естественным образом каждому участнику экономических отношений. Поэтому гармонизация промышленной и торговой политики предприятия видится как объективный, динамичный и реалистичный процесс. С точки зрения социальных и экономических отношений процессы по гармонизации значительно важнее, чем собственный поиск требуемого уровня гармонии. Также гармония важна, поскольку это эталон, или идеал, к которому следует стремиться участнику экономических отношений. Если отсутствует идеал, цель, то участник экономических отношений со значительной вероятностью впадет в так называемое состояние анергии (отсутствие желания и перспектив развиваться на предприятии).

Сегодня большое число научных публикаций посвящено исследованию эффективности, результативности, качества мероприятий промышленной и торговой политики предприятия. Гармонизация упоминается как следствие обеспечения эффективности производственной и торговой деятельности. На самом деле очень часто гармонизация предприятия, его действий, системы интересов обеспечивает расширение условий для совместимости элементов, всей системы развития производственной и торговой деятельности предприятия в целом. В данном случае гармонизация будет выступать как первичное понятие по отношению к качеству, уровню результативности и уровню эффективности мероприятий реализуемой промышленной, а также торговой политики на предприятии.

Иными словами, нахождение гармонии для предприятия, гармонизация должна рассматриваться как важная причина, а уровень качества, эффективности и результативности на предприятии - как следствия, которые характеризуют уровень гармонизации производственных и торговых процессов на предприятии. Важно отметить, что специфическим объектом для гармонизации промышленной и торговой политики будут выступать противоречия в производственных и торговых процессах на предприятии, противоречия интересов и действий предприятия, конкретизирующиеся посредством явлений (процессов), свойств (качества), целей (отношений). В отношении к целям промышленной и торговой политики гармонизация будет проявлять себя посредством постановки и согласования приоритетов, к качеству и свойствам производственных и торговых процессов как важное и необходимое условие их согласования, а к самим производственным и торговым процессам как мера, как критериальная база (например, степень автономности и зависимости производственных и торговых процессов). Фактически результатом гармонизации свойств производственных и торговых процессов будет выступать качество, гармонизации самих производственных и торговых процессов - эффективность, гармонизации целей промышленной и торговой политики - результативность производства и продаж продукции предприятия.

Таким образом, по своему содержанию и сути гармонизация промышленной и торговой политик предприятия это, с одной стороны, стратегический план производства и продаж, а с другой стороны, рабочий инструмент, который применяется для достижения, реализации стратегических планов. При этом такой инструмент имеет определенную реальную, а также количественную меру, обладает жесткой логикой, проявляющейся в ряде моделей по построению гармоничных и сбалансированных отношений по отношению к производству, продажами продукции на предприятии. В данном случае гармоничность производственных и торговых процессов - более 
совершенное и глубокое понятие, а гармонизация промышленной и торговой политики на предприятии - это построение системы гармоничных взаимодействий между всеми участниками производственных и торговых процессов предприятия. Следует отметить, что принцип гармоничности производственных и торговых процессов будет проявляться в необходимой и достаточное мере применительно к свойствам по обеспечению гармонии. Сюда, например, необходимо относить свойства резонансности, эмержентности, минимальности уровня затрат, инверсии, а также фрактальности для мероприятий реализуемой промышленной, торговой политики.

Сформулируем определение понятия гармонизации промышленной, а также торговой политики предприятия при учете фактора экономической безопасности, так как именно экономическая безопасность является важным результатом гармонизации. Экономическая безопасность на предприятии - достигнутый уровень (состояние) защищенности всех важнейших интересов предприятия применительно к внутренним и внешним угрозам, который формируется руководством и работниками предприятия посредством осуществления комплекса мероприятий экономического, организационного, правового, социально-психологического и инженернотехнического направлений, затрагивающих, в том числе, и вопросы организации производственной и торговой деятельности предприятия. При определении уровня экономической безопасности на предприятии главным выступает тот факт, что: уровень (состояние) защищенности обладает динамическим характером; внутренние угрозы также важны для рассмотрения, как и внешние угрозы; система по обеспечению экономической безопасности на предприятии в обязательном порядке взаимодействует на правовой базе с государством в вопросах обеспечения национальной безопасности в целом.

Экономическая безопасность на предприятии обеспечивается преимущественно действенностью организационных, материальных, нормативных гарантий в отношении установления, предупреждения (локализации) и пресечения возможных посягательств применительно к порядку управления, законным правам предприятия, к его имущественным активам, интеллектуальной собственности, позитивной финансовой, а также коммерческой конъюнктуре, высокому уровню устойчивости производственных и торговых процессов, социальной и психологической обстановке на предприятии, производственной дисциплине, различным научным достижениям и всей охраняемой качественной информации на предприятии.

Здесь управленческие гарантии - гарантии, создаваемые на основе разработки, а также поддержания высокого и требуемого уровня работоспособности для организационной структуры применительно к управлению процессами по выделению и устранению угроз для производственной и торговой деятельности на предприятии. Далее, ресурсные гарантии - гарантии, которые создаются на основе структуризации, рационального и эффективного применения комплекса ресурсов на предприятии с точки зрения своевременного установления, последовательного ослабления источников угроз, предотвращения (локализации) ущербов, расширение спектра условий, возможностей для реализации мероприятий производственной, торговой деятельности на предприятии. Как результат, правовые гарантии - гарантии по законодательному обеспечению производственной, торговой деятельности на предприятии, когда происходит формирование комплекса эффективных и юридически обоснованных взаимодействий с партнерами предприятия. Нормы права в данном случае определяют содержание режимов по обеспечению экономической безопасности на предприятии, что в случае их сочетания с рядом мер, связанных с установлением, поддержанием указанного режима обеспечит требуемый правовой базис всех действий системы безопасности на предприятии.

Необходимо понимать, что производственная и торговая деятельность современного промышленного предприятия предполагает решение широкого спектра организационных вопросов, правовых и экономических проблем, учет большого числа технических и кадровых аспектов. Особенно сложно эффективно управлять производственной и торговой деятельностью крупного промышленного предприятия, которое имеет широкий спектр деловых связей и большое число контрагентов: клиентов, поставщиков, кредиторов. Именно в рамках внешних и внутренних производственных и торговых связей предприятия возникают и реализуются угрозы для экономической безопасности предприятия. Здесь для обеспечения требуемой степени защиты предприятия от данных угроз возникает важность гармонизации промышленной и торговой политик предприятия, причем указанная активность должна обладать системным характером, в силу того, что объекты защиты определяют ключевые характеристики для системы по обеспечению экономической безопасности на предприятии. Так как производственная и торговая деятельности как объекты защиты очень сложны, имея значительное число параметров, в данном случае обеспечение требуемого уровня экономической безопасности следует рассматривать в комплексе с мероприятиями по гармонизации промышленной и торговой политик предприятия. 
Все это определило тот факт, что в научной литературе есть ряд определений понятия гармонизации промышленной и торговой политики предприятия, в том числе, и с учетом специфики обеспечения экономической безопасности. Например, под гармонизацией промышленной, торговой политик на предприятии с учетом обеспечения экономической безопасности предлагается понимать управляемую деятельность, которая позволяет повысить уровень социально-экономической эффективности, достичь необходимого консенсуса между разнонаправленными экономическими интересами различных субъектов внешней и внутренней среды, пропорционально согласовать разные направления производственной и торговой деятельности предприятия на базе координирующего центра подразделения стратегического маркетинга, как системы, которая затрагивает большинство составляющих элементов, важнейшим аспектом данной системы выступает гармонизация интересов используемых человеческих ресурсов на предприятии, повышение справедливости, ответственности предприятия за все совершаемые производственные и торговые действия [6]. С данным пониманием термина согласуется определение, данное Т. А. Жарковой, а именно: «гармонизация промышленной и торговой политики предприятия это «...комплексным образом согласованная и упорядоченная совокупность экономических процессов и элементов системы управления, образующая соразмерное соотношение и взаимовлияние промышленной и торговой деятельности ...» [5, с. 11].

В работе В. Н. Батовой [3]указывается, что проблему гармонизации мероприятий промышленной и торговой политики на предприятии следует считать важнейшей научной задачей стратегического менеджмента предприятия, так как правомерное и рациональное решение указанной проблемы обладает большим хозяйственным значением. В рамках реализации мероприятий по гармонизации промышленной и торговой политики на предприятии будет устанавливаться баланс значительного числа микроэкономических и макроэкономических факторов, которые определяют экономическую безопасность, возможности устойчивого развития, оптимальность рыночной стратегии предприятия, экономическую эффективность его проектов, уровень социальной ориентации производственной и торговой деятельности предприятия. Отсюда успешная производственная и торговая деятельность предприятия будут обуславливаться подходом к организации и управлению предприятием. Поэтому В. Н. Батова предлагает под гармонизацией мероприятий производственной и торговой деятельности на предприятии при учете фактора экономической безопасности понимать формирование эффективной и согласованной системы по производству и реализации продукции, работ, услуг, что в долгосрочной перспективе позволит создавать и расширять спектр условий для более эффективного и рационального применения резервов предприятия, выступает базисом для динамического и устойчивого развития предприятия, отраслевых и региональных экономик, функционирования национальной экономики в целом.

В статье Ю. А. Клейменовой [7] под процессом гармонизации торговой и промышленной политики на предприятии с учетом экономической безопасности предлагается понимать деятельность по балансированию экономических интересов предприятия, отдельных микроэкономических агентов, государства по поводу производства, реализации различных видов продукции, работ, услуг, что обеспечивает и производство продукции высокого уровня конкурентоспособности, и реализацию данной продукции на внутренних и мировых товарных рынках по критерию нахождения оптимальных условий для использования материальных и интеллектуальных ресурсов предприятия.

А. Н. Ахрамеев в работе [1] утверждает, что суть исследуемых процессов связана с выражением системы экономических отношений, рыночных механизмов построения взаимодействий субъектов внешнего и внутреннего окружения предприятия по критерию достижения рациональной и высокой сбалансированности процессов производства и торговли продукцией предприятия. Специфической особенностью определения А. Н. Ахрамеева следует считать тот факт, что в рамках гармонизации происходит регулирование отношений между субъектами, участвующими в реализации мероприятий промышленной и торговой политики на предприятии как фактора его устойчивого роста посредством мониторинга и стратегического контроля уровня экономической безопасности предприятия. В данном определении стратегическим инструментарием гармонизации мероприятий производственной и торговой деятельности предприятия при учете фактора экономической безопасности реализуется важная цель социальная и экономическая эффективность организации производственной и торговой деятельности предприятия, где функции скидок и расходов стремятся к своему минимуму, при этом функции доходов и уровня социальной защищённости и ответственности (предприятия, его работников, государства) к максимуму.

Все сказанное выше позволяет сформулировать наше определение процессов по гармонизации мероприятий промышленной, а также торговой политики на предприятии при учете фактора экономической безопасности: 
гармонизация мероприятий промышленной, торговой политики на промышленном предприятии в самом общем случае - процесс согласования целей, принципов, методов, критериев и показателей производственных, сбытовых и обеспечивающих их бизнес-процессов на всех этапах жизненного цикла политики, который за счет обеспечения экономической безопасности на этих этапах приводит к снижению экономического ущерба от производственной, сбытовой и иной деятельности предприятия и способствует оптимизации его экономического поведения.

Оценка и анализ гармонизации полного жизненного цикла промышленной и торговой политик предприятия с учетом обеспечения их экономической безопасности основываются на трех направлениях.

1. Предлагается количественный показатель степени гармонизации между параметрами: цели, задачи, принципы, методы, критерии и показатели, в рамках одной составляющей промышленной и торговой политики и ориентированный на повышение рентабельности и экономической безопасности предприятия. Оценка степени гармонизации параметров проводится методом парных сравнений.

2. Предлагается количественный показатель степени гармонизации между параметрами в целом составляющих промышленной и торговой политики и ориентированный на повышение рентабельности и экономической безопасности предприятия. Оценка степени гармонизации параметров проводится по модифицированному нами методу парных сравнений.

3. Предлагаются количественные показатели оценки качества выполнения этапов диагностики, формирования, реализации, регулирования, гармонизации и развития промышленной и торговой политики предприятия и их составляющих и ориентированные на повышение рентабельности и экономической безопасности предприятия.

Оценка экономической безопасности находится в обратной зависимости от экономического ущерба или потерь в стоимостном выражении, полученных в реализации угроз и их последствий по результатам реализации промышленной и торговой политики с учетом их гармонизации. На практике отнесение ущерба к той или иной составляющей промышленной или торговой политики представляется трудоемким и слабо формализуемым. Поэтому предлагается оценивать экономическую безопасность с помощью трех показателей: в целом по промышленной и торговой политике, а также по предприятию. Экономическая безопасность по предприятию определяется в зависимости от суммы ущерба по промышленной и торговой политике.

Существует несколько основных видов рентабельности предприятия: рентабельность активов; уровень рентабельности продукции; рентабельность продаж; рентабельность основных средств; рентабельность персонала; рентабельность инвестиций в предприятие; рентабельность собственного капитала. В нашем случае используются три показателя.

1. Рентабельность предприятия, равная процентному отношению показателя балансовой прибыли (равен разнице между полученной выручкой и себестоимостью включая организационные и управленческие затраты, но до вычитания налогов) к суммарной стоимости всех оборотных и внеоборотных активов, производственных мощностей и ресурсов. Если оценка рентабельности предприятия будет невысокой, то должны приниматься определенные управленческие меры для улучшения ситуации.

2. Рентабельность основных производственных фондов - это рентабельность средств труда, непосредственно, косвенно принимающих участие в процессах производства продукции, также эти средства труда не меняют своей первоначальной формы. Кроме этого, срок их использования должен превышать год, а сумма амортизации входит в себестоимость услуг или продукции. Рентабельность основных средств характеризует промышленную политику предприятия и показывает насколько эффективна экономическая деятельность бизнес-проекта и определяется как процентное отношение чистой прибыли к стоимости основных фондов. Коэффициент напрямую зависит от прибыльности и не должен быть меньше нуля: это означает, что предприятие работает с убытками и нерационально использует свои основные средства.

3. Рентабельность реализованной продукции рассчитывается как процентное отношение чистой прибыли к себестоимости. Полученный коэффициент помогает определить эффективность от реализации произведенной продукции и характеризует торговую политику предприятия.

Итак, исследование вопросов гармонии в экономике или в экономических отношениях раскрывают существующих антагонистических противоречий во внешней и внутренней средах деятельности участников экономических отношений. Наша трактовка понятия гармонии в экономических отношениях связана с установлением идеального динамичного и/или статичного состояния социально -экономической системы, которое характеризуется пропорциональностью процессов на этапах воспроизводственного цикла (производство, распределение, обмен, 
потребление, а также процессов упорядоченностью данных процессов, выбранной рациональной моделью специализации системы, максимальной согласованностью и сбалансированностью этих процессов, их соответствием требованиям внешней среды и друг другу по критерию единства социальной, экономической, экологической составляющих деятельности, выраженных соответствующими целевыми установками (системой ключевых показателей) по использованию интеллектуальных и материальных ресурсов системы.

По своему содержанию и сути гармонизация промышленной и торговой политик предприятия - это, с одной стороны стратегический план производства и продаж, а с другой стороны, рабочий инструмент для достижения стратегического плана.

Гармонизация промышленной и торговой политики промышленного предприятия в самом общем случае - процесс согласования целей, принципов, методов, критериев и показателей производственных, сбытовых и обеспечивающих их бизнес-процессов на всех этапах жизненного цикла политики, который за счет обеспечения экономической безопасности на этих этапах приводит к снижению экономического ущерба от производственной, сбытовой и иной деятельности предприятия и способствует оптимизации его экономического поведения.

Библиографический список

1. Ахрамеев, А. Н. Стратегический контроллинг гармонизации промышленной и торговой политики субъектов малого бизнеса: автореф. дис. ... канд. экон. наук: 08.00.05. - М.: МГУПП, 2014. - 28 с.

2. Бастиа, Ф. Экономические гармонии. Пер. с фр. - М.: Эксмо, 2007. - 1196 с.

3. Батова, В. Н. Гармонизация торгово-промышленной политики предприятия с учетом экономической безопасности // Гуманитарные, социально-экономические и общественные науки - 2012. - № 5. - С. 344-347.

4. Даль, В. И. Толковый словарь [Электронный ресурс]. - Режим доступа: https:/gufo.me/dict/dal/гармония (дата обращения: 13.03.2018).

5. Жаркова, Т. А. Обеспечение гармонизации промышленной и торговой политики на примере автомобильной корпорации: автореф. канд. экон. наук: 08.00.05. - М.: СОПС, 2015. - 26 с.

6. Журавлев, А. И. Вопросы гармонизации торговой и промышленной политики // Внешняя торговля. - 2009. - № 4-6. С. 25-32.

7. Клейменова, Ю. А. Гармонизация промышленной и торговой политики как фактор модернизации экономики // Вестник ЮРГТУ (НПИ). - 2012. - № 3. - С. 146-149.

8. Муратов, А. С. Гармонизационный подход к экономике и управлению предприятиями: теория и практика: монография / А. С. Муратов. - М.: Креативная экономика, 2011. -385 с.

\section{References}

1. Akhrameev, A. N. Strategicheskiy kontroling garmonizatsii promyshlennoy i torgovoy politiki subektov malogo biznesa: avtoref. dis. kand. ekon. nauk [Strategic controlling of harmonization of industrial and trade policy of subjects of small business: Abstract of thesis of Cand. Econ. Sci]. M.: MSUFP, 2014. 28 p.

2. Bastia, F. Ekonomicheskie garmonii [Economic harmonies / F. Bastia. Translation from French]. M.: Eksmo, 2007. 1196 p.

3. Batova, V. N. Garmonizatsiya torgovo-promyshlennoy politiki predpriatiya s uchetom ekonomicheskoy bezopasnosti [Harmonization of trade and industrial policy of the enterprise taking into account economic security] // Humanitarian, social and economic and social sciences, 2012, I. 5, pp. 344-347.

4. Dahl, V. Tolkovy slovar [Explanatory dictionary]. Available at: https://gufo.me/dict/dal/garmoniya. (accessed 13.03.2018).

5. Zharkova, T. A. Obespechenie garmonizatsii promyshlennoy i torgovoy politiki na primere avtomobilnoy korporatsii: Avtoref. dis. kand. econ. nauk [Ensuring of harmonization of industrial and trade policy on the example of an automobile corporation: Abstract of thesis of Cand. Econ. Sci]. M.: SSPF, 2015. 26 p.

6. Zhuravlev, A. I. Voprosy garmonizatsii torgovoy I promyshlennoy politiki [Questions of harmonization of trade and industrial policy] // Foreign trade, 2009, I. 4-6, pp. 25-32.

7. Kleymenova, Yu. A. Garmonizatsiya promyshlennoy i torgovoy politiki kak factor modernizatsii ekonomiki [Harmonization of industrial and trade policy as an economy modernization factor] // BulletinSRSPU (NPI), 2012, I. 3, pp. 146-149.

8. Muratov, A. S. Garmonizatsionny podkhod k ekonomike i upravleniyu predprayatiyami: teoriya i praktika: monografiya [Garmonizing approach to economy and management of the enterprises: theory and practice: monograph]. M.: Creative economy, 2011. 385 p. 